\title{
TOLERÂNCIA DE CANA-DE-AÇÚCAR À APLICAÇÃO SEQÜENCIAL DE NEMATICIDAS E HERBICIDAS
}

\author{
José Carlos Rolim ${ }^{1}$, José Claudionir Carvalho² ${ }^{2}$ Wilson R. T. Novaretti ${ }^{3}$ e Luís R. Voss ${ }^{4}$
}

\author{
${ }^{1}$ Engo . Agrônomo, Dr., Professor. Universidade Federal de São Carlos, Centro de Ciências Agrárias. Caixa Postal 153. \\ Araras, SP, 13600-970 jerolim@cca.ufscar.br \\ ${ }^{2}$ Eng ${ }^{\circ}$. Agrônomo, Dr., Pesquisador. Rohm and Haas Química Ltda. Paulínia, SP \\ ${ }^{3}$ Eng ${ }^{\circ}$. Agrônomo, Dr. Laboratório A.N.N.A. Piracicaba, SP \\ ${ }^{4}$ Eng $^{\circ}$. Agrônomo, Usina Equipav. Promissão, SP
}

\begin{abstract}
RESUMO
Um experimento foi instalado em solo arenoso (85\% de areia) na Usina Equipav, Promissão-SP, com o objetivo de estudar a possibilidade de ocorrência de interação entre nematicidas e herbicidas aplicados em seqüência, na cultura da canade-açúcar, variedade SP81-3250. O delineamento experimental adotado foi o de blocos ao acaso, com 4 repetições, no esquema fatorial, com 3 níveis da variável 1 (nematicidas) e 5 níveis da variável 2 (herbicidas). A aplicação dos nematicidas terbufós e carbofuran, ambos a $3000 \mathrm{~g} / \mathrm{ha}$, foi realizada manualmente, no fundo do sulco de plantio. Um outro tratamento foi utilizado, sem a aplicação de nematicida. Os herbicidas aplicados em pré-emergência da cultura e das plantas daninhas, foram : oxyfluorfen+ametryne $(480+1500 \mathrm{~g} / \mathrm{ha})$, oxyfluorfen+diuron $(480+2400 \mathrm{~g} / \mathrm{ha})$ e thiazopyr+ametryne $(360+1500 \mathrm{~g} /$ ha), além das testemunhas com e sem capina. As chuvas freqüentes ocorridas no primeiro mês do experimento proporcionaram uma situação interativa bastante drástica entre os produtos. Concluiu-se que não existiu interação significativa entre os nematicidas e herbicidas, que viesse a interferir na produtividade da cultura da cana-de-açúcar. O carbofuran conduziu a uma produtividade agrícola maior do que o terbufós, e este maior do que a testemunha; thiazopyr+ametryne causou no início os maiores sintomas visuais de fitotoxicidade, os quais não foram responsáveis por quebra de produtividade agrícola; oxyfluorfen+ametryne e oxyfluorfen+diuron, na ausência ou na presença de carbofuran ou terbufós, comportaram-se como os produtos mais seletivos para a cultura da cana-de-açúcar.
\end{abstract}

Palavras-chave: ametryne, diuron, oxyfluorfen, Saccharum spp., seletividade, thiazopyr.

\section{ABSTRACT \\ Sugar cane tolerance to sequential application of nematicides and herbicides}

A field experiment was carried out in a sandy soil (85\% of sand) aiming at studying herbicide/nematicide interaction in sugar cane. Terbufos and carbofuran, both applied at $3000 \mathrm{~g} / \mathrm{ha}$ in the furrow at planting and the herbicides oxyfluorfen+ametryne $(480+1500 \mathrm{~g} / \mathrm{ha})$, oxyfluorfen+diuron $(480+2400 \mathrm{~g} / \mathrm{ha})$ and thiazopyr+ametryn $(360+1500 \mathrm{~g} / \mathrm{ha}) \mathrm{were}$ studied. The herbicides were applied before emergence of the sugar cane plants, with a $\mathrm{CO}_{2}$ knapsack sprayer, equipped with 6 nozzles Teejet DG 80.02. The experimental design was a completely randomized block design in a factorial $3 \times 5$ arrangement, with four replications. The nematicides did not show any interaction with the herbicides. Carbofuran was better than 
José Carlos Rolim et al.

terbufós, producing higher sugarcane yield. Thiazopyr + ametryn showed the most severe phytotoxic effects until 123 DAA (days after application). A tank mixture oxyfluorfen + ametryn and oxyfluorfen+diuron were the most selective products for the sugarcane plants. Sugarcane yield was not affected by any of the applied herbicides.

Key words: ametryne, diuron, oxyfluorfen, Saccharum spp., selectivity, thiazopyr.

\section{INTRODUÇÃO}

A possibilidade de ocorrência de interações com efeitos negativos à cultura quando se faz a aplicação de dois ou mais produtos fitossanitários, com ações isoladas bem definidas e diferenciadas, já foi comprovada em algumas situações.

Hammil \& Penner (1973 a,b,c), observaram a existência de interações entre os herbicidas alachlor, chlorbromuron e butylate com o nematicida carbofuran nas culturas de cevada e milho. Penner \& Early (1973) também estudaram o efeito destes herbicidas na distribuição e no metabolismo do carbofuran nestas culturas. Smith \& Tugwell (1975) detectaram a existência de interação entre propanil e carbofuran na cultura do arroz.

Na cultura da cana-de-açúcar, Blanco et al. (1980) foram os primeiros a relatar a ocorrência de sintomas de fitotoxicidade causados pela aplicação de carbofuran seguido de tebuthiuron. Em trabalho posterior, Blanco et al. (1983) determinaram que a interação entre tebuthiuron, aplicado nas doses de 0,80 e $1,20 \mathrm{~kg} /$ ha e carbofuran, na doses de 1,875 e $2,625 \mathrm{~kg} / \mathrm{ha}$ foi responsável pela redução da biomassa seca da parte aérea e das raízes da cana-de-açúcar. Coletti et al.(1983) também estudaram este assunto, detectando que o carbofuran aplicado na dose de $3,0 \mathrm{~kg} / \mathrm{ha}$ demonstrou interação com a mistura de ametryne+tebuthiuron $(1,60+0,96$ $\mathrm{kg} / \mathrm{ha})+2,4-\mathrm{D}(1,44 \mathrm{l} / \mathrm{ha})$, causando sintomas de fitotoxicidade na cana-de-açúcar, que foram classificados pelos autores como leves a moderados.

Com o advento de novos herbicidas e nematicidas para a cana-de-açúcar, é importante que seja estudada a possibilidade de interações entre eles, para melhor e mais rápida orientação aos produtores.
O objetivo deste trabalho foi estudar a interação entre os nematicidas terbufós e carbofuran e os herbicidas oxyfluorfen (aplicado em misturas de tanque com ametryne ou diuron) e thiazopyr em mistura com ametryne, na cultura da cana-de-açúcar.

\section{MATERIAL E MÉTODOS}

O experimento foi instalado em Latossolo VermelhoAmarelo, textura arenosa, eutrófico, localizado na Usina Equipav, Promissão/SP, em relevo ondulado, com declividade menor que $3 \%$, contendo $15 \%$ de argila, $85 \%$ de areia e com teor de matéria orgânica de $10 \mathrm{~g} / \mathrm{dm}^{3}$. A área experimental foi previamente preparada destruindo-se a soqueira da cultura anterior, através da aplicação de glyphosate, na dose de $1800 \mathrm{~g} / \mathrm{ha}$, e, em seguida, a área foi calcareada com 2,0 t/ha de calcário dolomítico. Os sulcos de plantio foram abertos mecanicamente no dia 19/02/1998 e, na seqüência, procedeu-se a aplicação dos tratamentos nematicidas, manualmente, no fundo do sulco de plantio, na dose de $3000 \mathrm{~g} / \mathrm{ha}$ de terbufós ou de carbofuran, tendo sido utilizado um outro tratamento sem a aplicação de nematicida. Foi aplicado o inseticida fipronil, na dose de $0,25 \mathrm{~kg} / \mathrm{ha}$, em todos os sulcos de plantio, para controle de cupins subterrâneos. O plantio foi efetuado a seguir, tendo sido utilizada a variedade SP813250, com mudas de 11 meses, no espaçamento de 1,4 metros, com adubação fluida de plantio de 1000 l/ha da fórmula 0210-05 adicionada de $300 \mathrm{~kg} / \mathrm{ha}$ de Fosmag 464. Logo após a cobertura dos sulcos fez-se a aplicação dos herbicidas, em condições de pré-emergência da cana-de-açúcar e das plantas daninhas, trabalhando-se com equipamento costal pressurizado $\left(\mathrm{CO}_{2}\right)$, provido de barra com 6 bicos Teejet DG

Tabela 1. Características dos produtos utilizados no experimento. Promissão-SP. 1998/99.

\begin{tabular}{lccc}
\hline \multicolumn{1}{c}{ Nome Técnico } & Nome Comercial & Concentração & Formulação \\
\hline Oxyfluorfen & Goal & $240 \mathrm{~g} / \mathrm{l}$ & Concentrado emulsionável \\
Ametryne & Gesapax & $500 \mathrm{~g} / \mathrm{l}$ & Suspensão concentrada \\
Diuron & Karmex & $500 \mathrm{~g} / \mathrm{l}$ & Suspensão concentrada \\
Thiazopyr & Visor & $240 \mathrm{~g} / \mathrm{l}$ & Concentrado emulsionável \\
Terbufós & Counter & $50 \mathrm{~g} / \mathrm{kg}$ & Granulado \\
Carbofuran & Furadan & $50 \mathrm{~g} / \mathrm{kg}$ & Granulado \\
\hline
\end{tabular}

Fonte: Rodrigues \& Almeida, 1998; Andrei, 1999 
80.02 VS, espaçados de $50 \mathrm{~cm}$, perfazendo largura de faixa de aplicação de 3 metros, com pressão de $3,6 \mathrm{~kg} / \mathrm{cm}^{2}$ e consumo de calda de $200 \mathrm{l} / \mathrm{ha}$. Os tratamentos herbicidas, com suas respectivas doses $(\mathrm{g} / \mathrm{ha})$ foram : oxyfluorfen+ametryne $(480+1500)$, oxyfluorfen+diuron $(480+2400)$ e thiazopyr+ametryne $(360+1500)$. Tratamentos sem herbicidas, foram mantidos no limpo através de capinas manuais. As características dos produtos utilizados no experimento constam na Tabela 1 . O delineamento experimental aplicado foi o de blocos ao acaso no esquema fatorial 3 × 5 ( 3 nematicidas e 5 herbicidas), com 4 repetições. Cada parcela foi constituída de 5 sulcos de cana-de-açúcar de 10 metros de comprimento, sendo considerada como útil a área delimitada pelas 3 linhas centrais. Os dados obtidos foram submetidos à análise de variância pelo teste $\mathrm{F}$ e as médias comparadas pelo teste de Tukey a $5 \%$ de probabilidade.

Os sintomas de fitotoxicidade na cultura foram determinados aos 32, 89 e 123 DAT (dias após tratamento), segundo escala de notas relativas ao percentual de redução da biomassa aérea, onde a nota zero corresponde a nenhuma redução da parte aérea e a nota 100 corresponde a $100 \%$ de redução. A biometria das plantas foi estudada levando-se em conta o número de perfilho e a altura das plantas até o "dew lap" aos 96 e 150 DAT. A população de nematóides nas raízes das plantas e no solo foi determinada aos 106 DAT. Por ocasião da colheita foi analisada a produtividade da cana-deaçúcar.

\section{RESULTADOS E DISCUSSÃO}

Cerca de $190 \mathrm{~mm}$ de chuvas ocorreram antes da instalação do experimento, sendo que $85 \mathrm{~mm}$ aconteceram 4 dias antes do plantio e aplicação dos produtos. Após o plantio, $201 \mathrm{~mm}$ foram registrados no $1^{\circ}$ mês do ensaio, tendo ocorrido a primeira chuva (de $17 \mathrm{~mm}$ ) 5 dias após esta data. As chuvas freqüentes ocorridas, aliadas ao fator solo arenoso, permitiram condições excelentes de estudo da interação entre os tratamentos herbicidas com os tratamentos nematicidas. Estas precipitações atuaram, sem dúvida, no incremento do aparecimento de sintomas visuais de fitotoxicidade bem definidos nas plantas de cana-de-açúcar, provocados pelos produtos utilizados.

Os sintomas visuais de fitotoxicidade, expressos em termos de percentagem de redução da biomassa aérea das plantas, avaliados aos 32, 89 e 123 DAT, podem ser visualizados na Figura 1. A mistura de thiazopyr+ametryne provocou o aparecimento de sintomas de fitotoxicidade mais visíveis, os quais foram desaparecendo com o decorrer do tempo; sintomas mais persistentes decorrentes da aplicação desta mistura foram encontrados na interação com terbufós. A ausência de nematicida provocou as maiores reduções percentuais de biomassa de cana-de-açúcar, agravadas ao longo do tempo, comprovando o efeito deletério da ação da população de nematóides presentes na área.
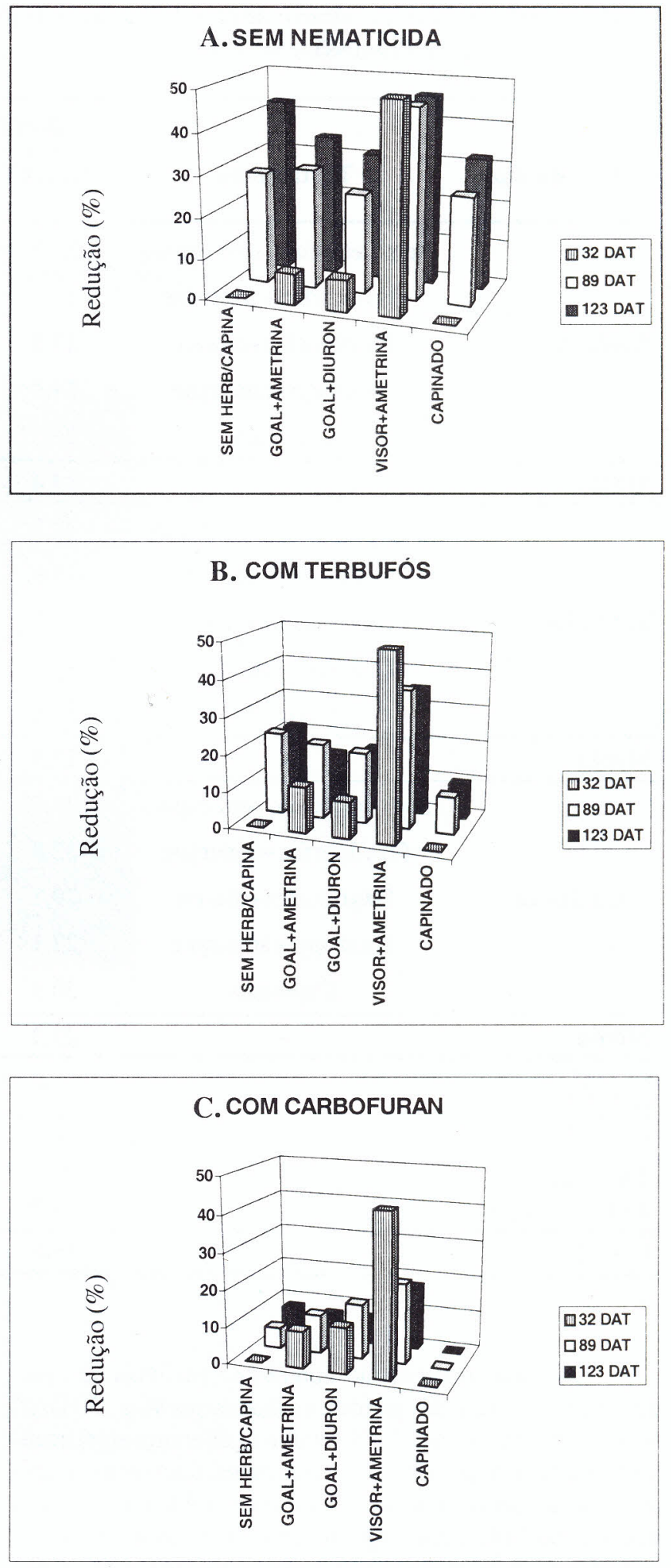

Figura 1. Representação visual dos sintomas de fitotoxicidade (\% de redução de biomassa de cana-de-açúcar) obtidos nas áreas: (A) sem nematicida, (B) com terbufós e (C) com carbofuran, aos 32, 89 e 123 DAT. Promissão, SP. 1998/99. 
José Carlos Rolim et al.

Tabela 2. Dados médios de número de perfilhos e altura de plantas aos 96 e 150 dias após o tratamento (DAT) e produtivida de agrícola. Promissão, SP. 1998/99.

\begin{tabular}{|c|c|c|c|c|c|c|}
\hline \multirow[b]{2}{*}{ Nematicida } & \multirow[b]{2}{*}{ Tratamento } & \multicolumn{2}{|c|}{ Perfilhos (n $/ \mathbf{m})$} & \multicolumn{2}{|c|}{ Altura (cm) } & \multirow{2}{*}{$\begin{array}{c}\text { Produtividade } \\
\text { (t/ha) }\end{array}$} \\
\hline & & 96 DAT & $150 \mathrm{DAT}$ & 96 DAT & 150 DAT & \\
\hline \multirow{5}{*}{ Ausência } & Sem herbicida, sem capina & 21,1 & 21,7 & 43,6 & 74,0 & 105,91 \\
\hline & Oxyfluorfen+ametryne & 27,8 & 28,2 & 40,9 & 67,0 & 129,71 \\
\hline & Oxyfluorfen+diuron & 23,5 & 26,2 & 41,8 & 66,5 & 127,33 \\
\hline & Thiazopyr+ametryne & 24,9 & 30,6 & 34,8 & 57,2 & 113,35 \\
\hline & Capinado & 24,5 & 28,7 & 42,5 & 68,0 & 117,22 \\
\hline Média & - & 24,4 & 27,1 & 40,7 & 66,5 & 118,70 \\
\hline \multirow{5}{*}{ Terbufós } & Sem herbicida, sem capina & 23,2 & 22,4 & 49,6 & 75,6 & 126,74 \\
\hline & Oxyfluorfen+ametryne & 29,9 & 29,4 & 44,6 & 72,3 & 121,68 \\
\hline & Oxyfluorfen+diuron & 28,9 & 32,4 & 46,4 & 78,9 & 133,58 \\
\hline & Thiazopyr+ametryne & 26,7 & 34,9 & 40,2 & 68,4 & 129,71 \\
\hline & Capinado & 31,0 & 31,0 & 48,4 & 74,5 & 127,03 \\
\hline Média & - & 27,9 & 30,0 & 45,8 & 73,9 & 127,75 \\
\hline \multirow{5}{*}{ Carbofuran } & Sem herbicida, sem capina & 26,5 & 24,1 & 57,2 & 84,0 & 129,11 \\
\hline & Oxyfluorfen+ametryne & 32,8 & 35,4 & 52,3 & 91,5 & 145,77 \\
\hline & Oxyfluorfen+diuron & 29,1 & 32,9 & 54,1 & 81,8 & 132,09 \\
\hline & Thiazopyr+ametryne & 27,1 & 31,0 & 45,6 & 75,8 & 143,10 \\
\hline & Capinado & 30,3 & 32,4 & 56,1 & 83,5 & 141,01 \\
\hline Média & - & 29,2 & 31,2 & 53,1 & 83,3 & 138,22 \\
\hline $\mathrm{F}_{\text {Nematicida }}$ & & $6,8 * *$ & $5,8 * *$ & $39,6 * *$ & $29,3 * *$ & $17,35 * *$ \\
\hline $\mathrm{F}_{\text {Herbicida }}$ & & $4,1 * *$ & $11,6 * *$ & $9,2 * *$ & $4,5 * *$ & 2,28 n.s. \\
\hline $\mathrm{F}_{\mathrm{NXH}}$ & & 0,3 n.s. & 1,1 n.s. & 0,1 n.s. & 1,1 n.s. & 1,93 n.s. \\
\hline d.m.s. Nematicida & & 3,2 & 2,9 & 3,3 & 5,3 & 8,05 \\
\hline d.m.s. Herbicida & & 4,9 & 4,5 & 5,1 & 8,0 & 12,21 \\
\hline C.V. (\%) & & 15,6 & 13,1 & 9,4 & 9,3 & 8,2 \\
\hline
\end{tabular}

Os dados relativos ao número de perfilhos de canade-açúcar e a altura das plantas, avaliados aos 96 e 150 DAT, encontram-se na Tabela 2. Observou-se diferença significativa no número de perfilho dos tratamentos, tanto entre as áreas tratadas com nematicidas como com herbicidas. Entre os nematicidas, diferenças significativas no estande foram observadas entre as áreas tratadas com nematicidas e a área não tratada, que apresentou menor número de perfilho por metro nas duas avaliações efetuadas, não existindo diferenças significativas entre os dois nematicidas.

Entre os herbicidas, ocorreram diferenças significativas entre as áreas onde houve controle das infestantes (com herbicidas ou com capina) e a área testemunha, a qual apre- sentou menor número de perfilho nas duas épocas avaliadas. A não existência de diferença significativa na interação $\mathrm{N} \mathrm{x}$ $\mathrm{H}$ indicou que todos os herbicidas estudados tiveram o mesmo comportamento (relativo ao número de perfilhos) na ausência ou na presença do nematicida empregado.

Este foi o parâmetro que acusou a existência de diferenças significativas entre os tratamentos estudados. Assim, nas duas avaliações efetuadas, observou-se que a área sem nematicida apresentou as plantas de menor porte e que dentre os nematicidas estudados, o que proporcionou maior altura de colmos foi o carbofuran.

Dentre os herbicidas, o tratamento que revelou a menor altura de colmos (o que indica também sua fitotoxicidade) 
foi a mistura thiazopyr+ametryne, que diferiu significativamente de todos os demais tratamentos herbicidas. Não houve interação significativa entre $\mathrm{N}$ x $\mathrm{H}$, o que demonstrou que todos os herbicidas tiveram comportamento semelhante (relativo à altura das plantas) frente aos nematicidas estudados.

Para a produtividade agrícola, o teste $\mathrm{F}$ acusou a existência de diferenças significativas, ao nível de $1 \%$ de probabilidade, entre os nematicidas estudados. Da mesma forma, existiram diferenças significativas, ao nível de $10 \%$ de probabilidade, entre os herbicidas aplicados. Não ocorreu interação significativa entre nematicidas e herbicidas ( $\mathrm{N} \mathrm{x}$ $\mathrm{H}$ ), o que indicou que todos os tratamentos herbicidas se comportaram de modo similar frente aos tratamentos nematicidas efetuados.

De uma forma geral, ocorreram diferenças significativas entre as populações dos nematóides estudados -Pratylenchus zeae na raiz e no solo, Helicotylenchus spp. e Paratrichodorus spp., ambos no solo -- somente entre as áreas tratadas com carbofuran e terbufós e a área sem aplicação de nematicida, não existindo diferença significativa entre os nematicidas testados. Não foram observadas também diferenças significativas entre os herbicidas testados, com referência a estes parâmetros.

O que se constatou é que, na média, o carbofuran conduziu a produtividades agrícolas $8,2 \%$ superiores ao terbufós e que carbofuran e terbufós produziram, respectivamente, 16,4 e 7,6\% mais do que a testemunha, sem aplicação de qualquer nematicida. As misturas de oxyfluorfen+ametryne e oxyfluorfen+diuron produziram sensivelmente mais do que a testemunha não capinada, apresentando produtividade semelhante à testemunha capinada, fato que comprova a seletividade destas misturas de herbicidas para a cultura da cana-de-açúcar.

\section{CONCLUSÕES}

Não houve interação entre os nematicidas carbofuran e terbufós e os herbicidas oxyfluorfen+diuron, oxyfluorfen+ametryne e thiazopyr+ametryne, aplicados em mistura em tanque, que viesse a interferir na produtividade da cultura da cana-de-açúcar, variedade SP81-3250. A mistura thiazopyr+ametryne mostrou os maiores sintomas visuais de fitotoxicidade, os quais não foram suficientes para causar quebra de produtividade agrícola; o oxyfluorfen em mistura com diuron ou ametryne, na ausência ou na presença de carbofuran ou terbufós, comportaram-se como os produtos mais seletivos para a cultura. O carbofuran conduziu à produtividade agrícola maior do que o terbufós e este maior do que a testemunha sem tratamento nematicida.

\section{LITERATURA CITADA}

ANDREI, E. (Coord.). Compêndio de defensivos agrícolas. 6. ed. São Paulo: Andrei Editora, 1999. 672 p.

BLANCO, H.G.; COLETTI, J.T.; MATTOS, A. O. ; OKUNO, M.S. Fitotoxicidade em cana- de-açúcar causada pela interação de inseticida e herbicida residual. Biológico, v.46, n. 10, p. 235-240, 1980.

BLANCO, H.G.; OLIVEIRA, D.A.; COLETTI, J.T.; TERAO, D. Fitotoxicidade em cana-de-açúcar (Saccharum spp.) em solo arenoso, induzida pela interação de tebuthiuron e carbofuran. Biológico, v. 49, n. 9/10, p. 227-236, 1983.

COLETTI, J.T.; NOVARETTI, W.R.T.; MELLO FILHO, A. T.; GASPARINI, C.T. Diagnóstico de fitotoxicidade em cana-de-açúcar através da análise foliar. Boletim Técnico Copersucar. Piracicaba/SP. p. 26-31. 1983.

HAMMIL, A.S. ; PENNER, D. Interaction of alachlor and carbofuran. Weed Science, v. 21, n. 4, p. 330-335, 1973 a.

HAMMIL, A.S. ; PENNER, D. Chlorbromuron - carbofuran interaction in corn and barley. Weed Science, v. 21, n. 4, p. 335-338, 1973 b.

HAMMIL, A.S. ; PENNER, D. Butylaye and carbofuran interaction in barley and corn. Weed Science, v. 21, n. 4, p. 339-342. 1973 c.

PENNER, D. ; EARLY, R.W. Effect of alachlor, butylate and chlorbromuron on carbofuran distribution and metabolism in barley and corn. Weed Science, v. 21, n. 11, p. 360-62. 1973 .

RODRIGUES, B. N; ALMEIDA, F. S. Guia de herbicidas. 4. ed. Londrina, PR: Edição dos Autores, 1998.

SMITH, R.J.; TUGWELL, N.P. Propanil - carbofuran inter ction in rice. Weed Science, v. 23, n. 3, p. 176-178, 1975. 
Revista Brasileira de Herbicidas, v.2, n.3, 2001. 